Like antisense oligonucleotides, siRNAs use sequence complementarity to target an mRNA for destruction. Unlike the antisense pathway, the RNAi pathway couples the specificity of an RNA guide to the stability and efficiency of a multipleturnover protein enzyme.

Assembly of an siRNA strand into a RISC seems to protect it from rapid degradation, the normal fate of small single-stranded RNA in cells. With this durability in mind, Song et al. set out to test whether direct infusion of siRNAs into mice might protect them from fulminant hepatitis. Both mice and humans with this disease suffer severe hepatic failure, with consequent encephalopathy, cerebral edema, metabolic imbalance and organ collapse. Fulminant hepatitis leads to death in over twothirds of patients who do not receive liver transplants.

Song et al. chose as their target a cell surface receptor, Fas, required for cell death in the liver. Fas mediates cell death by both apoptotic and necrotic pathways ${ }^{5}$. A variety of experimental insults can trigger Fas-dependent hepatic cell death in mice. For example, exposure to concanavalin A triggers cell death and fulminant hepatitis.

It is not surprising, then, that blocking Fas expression or function can prevent death of liver cells, a finding borne out by several studies. An antisense oligonucleotide directed against Fas mRNA can abrogate fulminant hepatitis caused by agonistic Fas-specific monoclonal antibodies and mitigate acetaminophen-induced hepatitis ${ }^{6}$. Antibody neutralization of Fas ligand alleviates hepatitis B-induced chronic hepatitis in mice ${ }^{7}$. In addition, patients with fulminant hepatitis have higher levels of hepatic Fas expression and circulating soluble Fas ligand ${ }^{8}$. Together, these data suggest that reducing Fas will provide therapeutic benefit in humans.

Song et al. pretreated mice with Fas siRNA and then induced fulminant hepatitis by exposing the mice to concanavalin A or agonistic Fas-specific antibodies. They found that siRNA treatment blocked the development of fulminant hepatitis and improved survival-even if siRNA was delivered after initiating hepatic insult. Thus, reduction of Fas expression early in the course of hepatitis could prevent its progression to more virulent disease.

In these studies, Fas expression returned to normal levels 20 days after siRNA treatment. This finding offers encouragement that siRNA directed to the Fas gene might be used safely, as Fas-mediated apoptosis regulates the production of lymphocytes. Mice lacking either Fas or Fas ligand develop a lymphoproliferative syndrome, and patients with dominant-negative Fas mutations have autoimmune lymphoproliferative disease $^{9}$. Long-term silencing of Fas could therefore have deleterious consequences, and the risks of even a temporary reduction in Fas will need to be monitored.

As therapeutic agents, siRNAs have enticing properties. Their actions appear to be short-lived in mammals; they are sequence specific; and they are natural, cellular products and may therefore not produce toxic metabolites. Nonetheless, caveats for clinical use remain. Delivering siRNAs to the appropriate cells is a major challenge. siRNAs have thus far only been administered intravenously to mice by 'hydrodynamic transfection,' the rapid infusion of siRNA in a volume one-tenth the mass of the animal. Furthermore, the liver seems to be particularly receptive to exogenous RNA. Better delivery methods-such as formulation of siRNAs with compounds that promote transit across cell membranes-are clearly required before siRNAs can be used in therapy, especially to suppress gene expression in tissues other than the liver. Nonetheless, the results of Song et al. begin to reveal the power of siRNAs in a disease model.

1. Song, E. et al. RNA interference targeting Fas protects mice from fulminant hepatitis. Nat. Med. 9, 347-351.

2. Elbashir, S.M., Lendeckel, W. \& Tuschl, T. RNA interference is mediated by 21 - and 22-nucleotide RNAs. Genes Dev. 15, 188-200 (2001).

3. Plasterk, R.H. RNA silencing: the genome's immune system. Science 296, 1263-1265 (2002).

4. Pomerantz, R.J. RNA interference meets HIV-1: will silence be golden? Nat. Med. 8, 659-660 (2002).

5. Wajant, $\mathrm{H}$. The Fas signaling pathway: more than a paradigm. Science 296, 1635-1636 (2002).

6. Zhang, H. et al. Reduction of liver Fas expression by an antisense oligonucleotide protects mice from fulminant hepatitis. Nat. Biotechnol. 18, 862-867 (2000).

7. Nakamoto, Y. et al. Prevention of hepatocellular carcinoma development associated with chronic hepatitis by anti-Fas ligand antibody therapy. J. Exp. Med .196, 1105-1111 (2002).

8. Ryo, K. et al. Significance of Fas antigen-mediated apoptosis in human fulminant hepatic failure. Am. J. Gastroenterol. 95, 2047-2055 (2000).

9. Lenardo, M. et al. Mature T lymphocyte apoptosisimmune regulation in a dynamic and unpredictable antigenic environment. Annu. Rev. Immunol. 17, 221-253 (1999).

${ }^{1}$ Department of Biochemistry and

Molecular Pharmacology and

${ }^{2}$ Division of Endocrinology and Metabolism

Department of Medicine

University of Massachusetts Medical School

Worcester, Massachusetts, USA 01605

E-mail:neil.aronin@umassmed.edu and

phillip.zamore@umassmed.edu

\title{
Mend the gap
}

Bone is a tough substance, but exactly how it resists cracks and breaks has been difficult to determine. In the March of Nature Materials, Nalla et al. take on this question. One way that bones stay tough is by bridging gaps using structures such as collagen fibrils) (shown in this scanning electron micrograph). An even more important contributor to bone toughness is deflection of cracks along so-called cement lines, the boundaries between osteons (cylinder-like bone structures). The authors made notches on the edges of bones and found that the cracks developed similiarly to those seen in ductile substances such as certain metal alloys-at the site of the notch, instead of ahead of it. The investigators are eager to examine bone properties in people of different ages and with diseases such as osteoporosis. Some osteoporosis drugs can inhibit bone remodeling, a process that counterbalances bone consumption with bone formation. Such treatments could result in disrepair and the accumulation of 'micro-cracks' as the body ages.

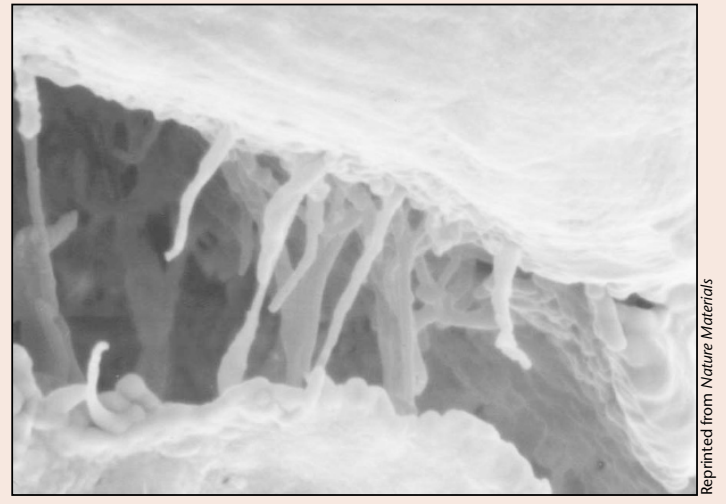

Charlotte SCHuberT 